\title{
Naturalezas, culturas y arquitecturas disciplinarias. La infrapolítica de un debate (necesariamente) interminable*
} Nature, culture and disciplinary architectures. Infrapolitics of an (inevitably) unending debate

\author{
Siobhan F. Guerrero Mc Manus ${ }^{\dagger}$
}

\begin{abstract}
Resumen
En este texto se analizan aquellas disciplinas, como la paleoantropología, la bioarqueología y la ecología humana, en las cuales confluyen diversos saberes, provenientes de las ciencias naturales, sociales y humanas. Estas ciencias son los puntos donde se negocian las relaciones entre estos agrupamientos, son los sitios donde se gestan los márgenes que delimitan a los saberes. De allí que sea importante analizar las dinámicas que rigen estos encuentros. En este texto se discute cómo esto ocurre dentro de proyectos arquitectónicos que buscan hacer coherentes a estos diversos cuerpos de saberes por medio de ciencias bisagra que se colocan a sí mismas como mediadoras. Sin embargo, como también se señala, este acto es inevitablemente político, pero en un sentido en el cual la política implica también una ponderación de ontologías en competencia a las cuales les subyacen dimensiones infrapolíticas de identificación y desidentificación. Finalmente, se señala que, si bien las fronteras entre lo humano y lo no humano son siempre negociables, no se debe perder de vista que las capacidades humanas para crear mundos no son simples instancias del proceso de construcción de nicho.
\end{abstract}

Palabras clave: naturaleza - cultura - filosofía de la interdisciplina

\begin{abstract}
In this text I analyze disciplines such as paleoanthropology, bioarcheology and human ecology in which different domains of knowledge intersect, some coming from natural sciences while others from social and human sciences. These disciplines are the hotspots in which the relationships among sciences are negotiated, in which the margins of science are drawn. Hence the relevance of analyzing the dynamics that direct these encounters. Here I argue how this occurs within the boundaries of an architectonical project that aims to connect in a coherent manner these different domains through "hinge sciences" that present themselves as mediators. However, I also show how this act of mediating is inescapably political although here politics always implies the ponderation of competing ontologies supported by infrapolitical dimensions ruled by processes of identification and dis-identification. Finally, I show why, even if the frontiers between the human and the nonhuman are always open to negotiation, we should not lose sight of the fact that our world-constructing capacities are not mere instances of niche construction.
\end{abstract}

Keywords: nature - culture - philosophy of interdisciplinarity

\footnotetext{
* Recibido: 26 de Febrero de 2016. Aceptado con revisiones: 5 de Diciembre de 2016.

+ Centro de Investigaciones Interdisciplinarias en Ciencias y Humanidades, Universidad Nacional Autónoma de México. Para contactar al autor, por favor, escribir a: FabrizzioMc@gmail.com.

Metatheoria 8(2)(2018): 77-85. ISSN 1853-2322.

(c) Editorial de la Universidad Nacional de Tres de Febrero. Publicado en la República Argentina.
} 


\section{Introducción}

En septiembre de 2015 los medios de comunicación de todo el mundo se volcaron a comentar un descubrimiento paleoantropológico que abría la posibilidad de reescribir -una vez más- la historia de la evolución humana. ${ }^{1}$ Me refiero por supuesto al barullo generado por Homo naledi, un homínido cuya morfología lo colocaba entre el grupo de los austrolopitecinos y especies propias del género Homo como vendría a ser Homo erectus.

La razón por la cual esta especie generó tal interés tiene que ver con el hallazgo de más de 1,500 fósiles en una sima al interior de una cueva. Pero estos fósiles no indicaban daño alguno así que la posible hipótesis de que estuviesen allí como resultado de una caída quedaba en entredicho. Más bien, se dijo, todo indicaba que estábamos ante el primer ritual funerario de la historia. Y, si esto era así, entonces nos estábamos confrontando con la asombrosa posibilidad de que estos elementos culturales tan característicos del humano moderno fueran mucho más antiguos de lo esperado.

Comienzo con esta historia por dos razones. Primero, porque ejemplifica un rasgo de un conjunto de disciplinas que se resisten a ser fácilmente catalogadas como ciencias naturales o ciencias sociales ejemplos adicionales a la paleoantropología lo serían disciplinas como la bioarqueología o la ecología humana- ya que justamente problematizan la coherencia misma de la distinción entre naturaleza y cultura o, quizás, porque mapean los linderos en los cuales éstas se cruzan (Marks 2011). En estas ciencias resulta difícil distinguir metodológicamente si estamos ante ciencias de la explicación o de la comprensión o si dicha oposición tiene siquiera sentido cuando examinamos fenómenos que resisten la dicotomía naturaleza vs cultura. Asimismo, estas disciplinas nos permiten examinar las arquitecturas de los saberes, no tanto a su interior, sino como parte de un sistema de conocimientos en el cual las disciplinas no son ni absolutamente modulares ni completamente intertraducibles o, siquiera, vinculables.

Segundo, más allá de la reflexión anterior, en la cual se intersectan elementos epistemológicos con elementos sociológicos, estas disciplinas nos permiten cuestionar seriamente el tipo de ontología que subyace a los objetos -sujetos- de estudio que estas disciplinas interrogan. En cierto sentido, no estamos aquí ante objetos cuyas propiedades se agoten por medio de descripciones físicas o químicas. Estos objetos -sujetos-, al estar vivos, están abiertos ante el mundo en ese sentido tan específico que Heidegger (1962) le dio al término "apertura", es decir, de un sujeto que es capaz de un saber sobre sí.

De hecho, es aquí donde aquellos episodios provenientes de las Guerras de las Ciencias y en las cuales se dieron fuertes discusiones en torno a nociones como el género, la orientación sexual, la raza, la inteligencia, etc. encontrarán cabida a modo de ejemplos que ilustran la tensión entre apuestas biologicistas que buscaron erigir arquitecturas reduccionistas fundadas en la biología, por un lado, y arquitecturas que resistieron esto último y buscaron defender la autonomía -tanto ontológica como epistemológica- de las ciencias sociales y humanas.

A modo de ejemplo, pensemos cómo el discurso que proviene de los estudios de género toma aquí relevancia ya que si en algo se ha insistido en este campo es en señalar que los géneros (hombre, mujer, genderqueer, etc.) propios del ser humano no sólo son irreductibles a la categoría de sexo, de corte biológico, sino que son ellos mismos el resultado de ser un sujeto embebido en un mundo cuya constitución demanda una noción robusta de cultura; esto es, una noción por principio irreductible a fenómenos en los cuales la significatividad se elimine. De allí que sea imposible describir a este tipo de propiedades como si lidiásemos con objetos pues estas propiedades sólo emergen cuando alguien se vive a sí mismo bajo esta lógica y ello demanda una noción de mundo de corte hermenéuticofenomenológico (Marder 2014). Es decir, lidiamos aquí con existenciarios y no con propiedades, con modos de ser y no con atributos de un ente (Heidegger 1962).

Por ello es que ese mundo no es solamente un compendio de objetos y relaciones sino que incluye

\footnotetext{
${ }^{1}$ Véase por ejemplo la siguiente nota: http://elpais.com/elpais/2015/09/09/ciencia/1441800892_046663.html (consultada por última vez el 26 de febrero de 2016).
} 
los efectos que tales entidades generan en él. En el caso humano -o, quizás, del grueso del género Homo- esta apertura ante el mundo y esta capacidad de intervenirlo parecen alcanzar un punto de quiebre tras el cual emergen mundos ontológicamente enriquecidos por nuevas relaciones, por nuevas entidades -como las normas o el significado- y por instituciones de corte social que permiten hablar propiamente de una cultura. Y es aquí donde cabe una pregunta cuyas respuestas pueden abarcar desde el más radical de los antropocentrismos hasta el más radical de los naturalismos: esta doble ruptura, entre lo vivo y lo no vivo, entre lo humano y lo no humano, ies un artificio de nuestras divisiones conceptuales y disciplinarias o recupera una suerte de transiciones evolutivas (Maynard-Smith \& Eörs 2000) que demandan arquitecturas disciplinarias específicas?

En este texto pretendo abordar -brevemente- ambos puntos, tanto la arquitectura de las disciplinas como la ontología de estos objetos marginales -por habitar en los márgenes de los saberes y trazar justamente márgenes ${ }^{2}$ que los dividen y no ya por poco importantes- que una y otra vez nos confrontan con las limitaciones y potencialidades de nuestros discursos. Comienzo así por el primer punto y luego doy lugar a la reflexión ontológica. Termino con unas breves conclusiones en las cuales se explicita por qué es éste un texto que resulta relevante para comprender a aspectos tales como la relación entre las ontologías sociales y naturales que atraviesan a los estudios de género -o incluso los estudios críticos sobre la noción de raza-.

\section{Construyendo arquitecturas}

Estas ciencias de frontera o de la marginalidad, como las he llamado anteriormente, requieren un análisis que combine los elementos de la epistemología y de la sociología de la ciencia en sentido amplio (incluyendo por tanto a los estudios sociales y culturales de la ciencia). Esta combinación no puede llamarse propiamente epistemología social pues no sólo recupera la dimensión social del conocimiento $^{3}$ sino también aspectos o consecuencias del mismo que no suelen ser analizados por dicha disciplina. Me refiero, por ejemplo, a las consecuencias biopolíticas de los saberes.

Es por ello que he decidido hablar de las arquitecturas del conocimiento, resaltando con ello dos elementos: por un lado, la relación entre los contenidos, modelos, representaciones y teorías de los saberes y, por otro, la relación entre los cuerpos disciplinarios en los cuales se ven encarnados. Con respecto a esta última noción, lo que se busca es reclutar al aparato foucaultiano ${ }^{4}$ en torno a lo disciplinario, como una suerte de ortopedia que opera sobre los cuerpos y que conforma sujetos, para pensar a las disciplinas no sólo como aquello que se aprende y enseña en las universidades y otros centros de investigación sino, también, para concebir a las disciplinas como espacios sociales que moldean a los sujetos que las practican; que gestan, de esta forma, identidades y valores disciplinarios.

Lo que se buscaría señalar con este giro sería por tanto el hecho de que las relaciones teóricas o conceptuales vienen a constituir cosmovisiones justamente porque gestan disciplinas -en tanto saberes- que son capaces de estructurar las dimensiones infrapolíticas que orientan a los sujetos así disciplinados y que, por tanto, los arrojan a nuevas formas de construir e interpretar al mundo. Es decir, las disciplinas disciplinan sociedades al crear arquitecturas del conocimiento con una serie de imperativos de coherencia que habrán de gestar modos de ser-en-el-mundo, es decir, gestan cosmologías.

De allí que un análisis acerca de tales disciplinas marginales deba prestar atención no únicamente a cómo se articulan las relaciones entre naturaleza y cultura -o a cómo se desarticula dicha oposición- en

\footnotetext{
${ }^{2}$ Tomo este término de Derrida (2008) quien lo emplea justamente para analizar los márgenes de la filosofía como aquella zona en la cual la filosofía se va enmarcando y definiendo pero también, aunque suene paradójico, se va expandiendo justamente al ir cuestionando e incorporando lo que anteriormente se tomaba como un mero marco, una forma de plasmar un problema que podía presuponerse sin mayor discusión.

${ }^{3}$ Una dimensión que puede desdoblarse en los elementos sociales que están presentes en la justificación, en la heurística, en la crítica o diseminación de sesgos, etc.

${ }^{4}$ Busco así recuperar el proyecto arqueogenealógico emprendido por Foucault; véase, por ejemplo, Foucault $(1970,1972)$.
} 
términos conceptuales o explicativos sino a los efectos que ello tiene en, por un lado, las relaciones entre diversas disciplinas y, por otro, en la imagen que sobre la naturaleza (humana y no humana) se construye (Giffney \& Hird 2008).

Pero antes de seguir elaborando dicha noción es menester argumentar en favor de su relevancia. Para ello me concentraré en dos elementos que considero importantes para analizar dichos saberes marginales. Primero, abordaré la forma en la cual estos saberes se juegan en el plano de la unidad -o pluralidad- de los objetos/sujetos bajo estudio. Segundo, discutiré brevemente cómo ello da lugar a una discusión sobre la continuidad de métodos y estrategias explicativas -o su discontinuidad- como resultado justamente del tópico anterior.

\subsection{Unidad o pluralidad de los objetos/sujetos bajo estudio}

Cuando aquí hablo de unidad me refiero a la forma en la cual diversas disciplinas, tanto en ciencias sociales y humanas como en ciencias naturales, introducen una oposición entre Ego y Alter, ${ }^{5}$ entre aquello que es un objeto que también es sujeto y aquello que es meramente un objeto; entre un agente que se estudia y una cosa que se investiga. Empleo los términos Ego y Alter para hacer ver que esta demarcación no es inocente ya que esconde un proceso de identificación entre un sujeto que investiga e inquiere y que se identifica justamente con aquellos objetos del mundo que se le parecen -o que cree que se le parecen- mientras que, por otro lado, se des-identifica con objetos que juzga como diferentes y, quizás, menos interesantes y menos complejos.

Nótese que en este punto mi análisis no versa tanto acerca de la veracidad de las representaciones que se hacen entre Ego y Alter o el hecho mismo de que se coloquen a ciertos fenómenos en uno u otro lado de tal división. Lo que me interesa, más bien, es atender a los efectos de verdad que tienen las elecciones que gobiernan la creación de fronteras -o su disolución- cuando se enfatiza la unidad o la des-unidad entre la naturaleza y la cultura o entre lo humano y lo no humano. De allí que me parezca que estas ideologías de la unidad o la des-unidad se constituyan en una suerte de infrapolítica de los saberes, una política que les subyace, y que tendrá efectos en cómo nos posicionamos en y ante el mundo. Pero es claro que esta infrapolítica no nace ella misma de la nada, sino que está ya estructurada, al menos parcialmente, por el tipo de relaciones de des/identificación -es decir, por la infrapolítica ya constituida y no por la infrapolítica que habrán de constituir- que nos sitúan dentro de una imagen de "lo natural". 6

En cualquier caso, tal pareciera que gran parte de las ciencias sociales y humanas construyen la oposición entre Ego y Alter como coextensa con la oposición entre lo humano y lo no humano o, quizás, para hilar más fino, como aquellos fenómenos vinculados a los rasgos específicos del ser humano como productor de cultura y aquellos rasgos que versan acerca de los procesos causales y materiales que gobiernan a la naturaleza. Pero no siempre la identificación habrá de darse entre Ego como lo humano y Alter como la diferencia que nombra a la naturaleza; la historia del racismo es sin duda el mejor ejemplo de un uso de esa frontera que animalizó a muchos grupos humanos y los arrojó a los confines de Alter.

Algo semejante ocurriría con aquellos proyectos al interior de las ciencias biológicas que han apostado por una unidad que no hace distinciones importantes entre los fenómenos propios de lo humano y los fenómenos propios del resto del ¿reino?, ¿clado?, ¿familia? Ego fagocita a Alter o Alter consume a Ego. Lo primero ocurre -i.e., que Ego fagocite a Alter- cuando se expande lo que suele pensarse como privativo de lo humano de tal suerte que su esfera se amplía; por ejemplo, cuando los

\footnotetext{
${ }^{5}$ Quizás en este punto resulte peculiar la forma en la que empleo la oposición entre Ego y Alter al señalar que lo primero identifica a un sujeto pensante y lo segundo a un mero objeto. Sin duda que tradicionalmente la oposición entre Ego y Alter se hace entre sujetos, pero justamente mi punto -y del giro no humano en filosofia- es que esta aplicación tiene una suerte de antesala en la cual se ha distinguido ya entre el dominio de los sujetos y el dominio de los objetos. A la luz de tal distinción es que suele pensarse la alteridad como una relación entre conespecíficos humanos, lo cual suele supeditarla ante un sesgo terriblemente antropocéntrico que pierde de vista una alteridad más profunda que no se problematiza, i.e., la oposición entre lo humano como consciente de sí, como ser pensante, y lo no humano como mero objeto, como entidad. De allí que yo sostenga que el primer momento -analítico y no biográfico- de oposición entre Ego y Alter sea entre lo que se juzga como sujeto y lo que se juzga como objeto.

${ }^{6}$ Este último párrafo únicamente señala la dimensión genealógica de las propias infrapolíticas de los saberes.
} 
modelos de construcción de nicho sostienen que la construcción de cultura, la construcción de mundos es solamente una instancia de un proceso presente en todo ser vivo. Lo que allí vemos es precisamente un Ego que expande las fronteras de los objetos que son también sujetos (v.gr. Laland et al. 2011, Odling-Smee \& Kevin 2011, Schultz 2015).

Ello no sólo ocurre en casos como el ya mencionado sino en ciertas ramas del ambientalismo que expanden la noción de persona para incorporar a seres no humanos, que acuñan conceptos como el de "paciente moral" para justamente ampliar a la ética. Homo naledi adquiere la relevancia mediática ya mencionada precisamente porque implica una revisión de las fronteras de lo humano.

Por otro lado, Alter consume a Ego allí donde lo específico de lo humano se niega, se reduce o se minimiza. A modo de ejemplo pensemos en los muchos biologicismos en los cuales la cultura se ve reducida a la naturaleza o se le juzga como epifenoménica de procesos biológicos subyacentes. Sea ya el caso de la sociobiología (Wilson 2000) o de la psicología evolutiva, la oposición que importa no es ya entre lo humano y lo no humano sino entre aquello que gobierna a lo vivo y aquello que gobierna a lo no vivo.

A modo de breve intermezzo, quizás es pertinente mencionar que las reflexiones aquí ofrecidas irán cobrando mucha mayor relevancia a la luz de la nueva filosofía ambiental y en esta época que ha venido a nombrarse como Antropoceno. Diversas tradiciones científicas y filosóficas lo ejemplifican, como por ejemplo: la Evo-Devo, la Eco-Evo-Devo, la Teoría de los Sistemas en Desarrollo (DST), las ciencias de la complejidad, el ecosocialismo, el ecomarxismo, la ecología queer y el ecofeminismo, el giro ambiental en el realismo crítico y en el realismo especulativo, la teoría de construcción de nicho, etc. En todos estos casos se aboga por abandonar la dicotomía naturaleza vs cultura, por repensar los confines de Ego y Alter, pero en cada ocasión el proyecto se ejecuta de formas diferentes. Cada uno de estos ejemplos conlleva una arquitectura disciplinaria ligeramente diferente y anclada en una infrapolítica distinta.

De allí que no baste con señalar la urgencia de superar la oposición entre naturaleza y cultura sino que sea necesario hilar fino para analizar cómo es que se da dicha superación, cómo es que se construye la unidad y la continuidad, cómo se erige la arquitectura tras dicha superación y qué consecuencias acarrea todo lo anterior.

Claro está, los procesos de identificación y des-identificación que estoy señalando, como una suerte de infrapolítica del proceso de categorización, pueden operar a modo de mecanismos antiparalelos. Por un lado, disminuyendo la agencia, la especificidad del sujeto, para luego proyectarlo más allá de los confines de Homo sapiens mientras, de forma antiparalela, dicha agencia disminuida, dicha especificidad, se naturaliza. Así, se humaniza una región del mundo pero de forma simultánea se deshumaniza a lo humano. Esto no tiene que ser algo puramente negativo como lo ilustran los ejemplos del ambientalismo.

En todo caso, lo que me interesa no es apuntalar un conjunto de conceptos claros y precisos sino, más bien, a los procesos de (des-)identificación que subyacen a la construcción de una oposición entre una categoría a la que se pertenece y una categoría a la que no se pertenece. Es esta actividad de trazar márgenes y sus sustratos extrateóricos/infrapolíticos lo que me interesa.

\subsection{Continuidad o discontinuidad en los métodos y las disciplinas}

Ahora bien, esta unidad o pluralidad del objeto bajo estudio da lugar a una continuidad o discontinuidad de métodos de investigación y de estrategias explicativas. Asimismo, da lugar a una continuidad o discontinuidad que se traduce en diferencias políticas que van más allá de la epistemología. Puede, por ejemplo, demandar el estudio de los primates por medio de métodos que recuperen elementos propios de la entografía y, por otro lado, hacer posible un trato mucho más ético ante estos animales que los reconozca como sujetos de derechos.

Por ejemplo, la aspiración de superar los dualismos entre naturaleza y cultura o bien el afán de comprender al ser humano como un fenómeno bio-psico-social, material y simbólico, podría motivar 
proyectos reduccionistas o eliminativistas en los cuales un dominio es subsumido ante otro o, finalmente, articulado desde el punto de vista de una disciplina que se coloca a sí misma como central y capaz de dar cuenta de cómo Ego u Alter son posibles.

Y es por esta última posibilidad el que me ha parecido importante traer a cuenta el aparato foucaultiano ya que las relaciones entre las disciplinas no son únicamente teóricas, lo cual a estas alturas no es ninguna sorpresa. Pero tampoco basta señalar que hay otras dimensiones de análisis, como las financieras, las institucionales, etc. Hay un punto adicional que versa acerca del poder que tienen ciertas disciplinas para presentarse a sí mismas como disciplinas bisagra o articuladoras, capaces de gestar arquitecturas del conocimiento que conecten los diversos saberes y cuyo cometido es conciliar los distintos trazos.

Sin embargo, esas arquitectónicas operan más allá del reduccionismo o sus variantes, más allá del holismo y más allá del monismo metodológico. Operan al imponer sus procesos de (des-)identificación como condición de posibilidad de la coherencia entre disciplinas. De allí que sugiera nombrar a este proceso bajo el rótulo de la construcción de Arquitectónicas de la Coherencia.

Dichas arquitectónicas no serían inocentes. En principio, implicarían la jerarquización de los saberes pero no ya en términos epistemológicos u ontológicos sino en términos disciplinarios. Quizás en ningún lugar es esto más claro que en las disciplinas marginales pues es en éstas en donde se analizan las transiciones (¿evolutivas?, ¿de fase?) de tal suerte que se ofrecen visiones omnicomprensivas que proponen un orden natural-cultural que justamente busca reconciliar los mecanismos de identificación y des-identificación ya descritos y propios de diversos saberes. Mecanismos que, en cualquier caso, (d)escriben ontologías.

\section{2. (D)escribiendo ontologías. Historia y tiempo, discurso y materia}

Parecería que, tras haber dicho lo anterior, no habría forma de tomar partido dentro de la discusión acerca de qué ontología describe -o escribe- de forma más adecuada -o deseable- a las relaciones entre lo natural y lo cultural o entre lo humano y lo no humano. En un sentido esto es así. No hay posibilidad de ocupar una posición metateórica que no se vea a sí misma subsumida por la lógica de un debate en el cual toda enunciación esconde una infrapolítica y una dimensión disciplinaria asociada a cierta arquitectónica de la coherencia.

Empero, así como en política se habla sin aspirar a ocupar una posición privilegiada -o, al menos, así se entiende en gran parte del post-estructuralismo- así también aquí habremos de expresar y defender una posición que tendrá ella misma consideraciones infrapolíticas. No se pretende, por tanto, resolver dialécticamente el debate entre lo natural y lo cultural como si fuese posible arribar a un holismo que estuviera él mismo más allá de las tensiones propias del debate que lo engendró. Tampoco se pretende una visión objetivista e ingenua que afirme que estamos ante una mera reflexión ontológica u epistemológica. Como dice Marder (2014) aquí se encuentra la dimensión más crítica del proyecto fenomenológico como una apuesta no únicamente epistémica y ontológica sino también política acerca de cómo se nos manifiesta el mundo y cómo, al encontrarlo, nos vamos transformando mutuamente.

Así, lo que aquí busco es simplemente explicitar una serie de riesgos que están presentes cuando, de manera apresurada, se decreta el fin de las así llamadas Guerras de las Ciencias; cuando se decreta el fin de las tensiones entre lo natural y lo social. Desde mi punto de vista, por más deseable que sea esta superación, ella esconde las semillas de nuevas batallas si se asume que la superación de un debate implica la disolución de la infrapolítica que lo impulsó. Como yo lo veo, simplemente las dimensiones infrapolíticas son ineliminables y de allí que la resolución de tal controversia no pueda nunca llegar, no llegará plenamente.

Para mostrar esto quisiera dar un rodeo en el cual reclutaré a una serie de pensadores que me ayudarán en mi argumentación. Comienzo así trayendo a Donna Haraway (2007) quien sostiene, siguiendo a Bruno Latour (2002), que nunca hemos sido humanos. Y nunca lo hemos sido porque no 
existe una única naturaleza humana, presente en todo tiempo, espacio y cultura. En cada momento, nos hemos coproducido con el resto del mundo y con el resto de los seres vivos. De allí que nuestra ontología no sea únicamente temporal, al admitir un tiempo evolutivo y ontogenético, sino que sea, asimismo, histórica ya que no sólo está sujeta a un devenir sino que este devenir está siempre moldeado por las diversas relaciones materiales de las cuales participamos. En otras palabras, nos define una intermaterialidad que no sólo cambia con el tiempo sino que es históricamente específica.

Pero, cómo interpretar esta afirmación. Primero, parece desestabilizar a la oposición entre Ego y Alter y la forma en la cual ésta se emplea en la confección de juicios acerca de la unidad y la pluralidad, de la continuidad y discontinuidad. En esta primera lectura se niega que Ego y Alter tengan sentido porque el uno y el otro son recursos de su mutua constitución, una constitución histórica y siempre cambiante. Y, sin embargo, esta lectura no deja de tener efectos arquitectónicos y biopolíticos. Aquí ya no sería la biología la ciencia bisagra sino quizás cierto materialismo histórico devenido en filosofía de la naturaleza; aquí se coloca al mundo como un tejido, como un ente tejido -un entre-tejido- de interacciones materiales. Si la metáfora es textil y el mundo y la naturaleza se entretejen, entonces la intermaterialidad es el correlato materialista de la interdiscursividad, de la intertextualidad -y es, de hecho, su sustrato material-. Tejido y texto vienen aquí a interdefinirse en un sentido que rebasa sus propias etimologías comunes.

Pero sería allí, en ese punto de encuentro, donde habrá de gestarse un desencuentro. Si la afirmación Harawayana pretende desestabilizar la oposición entre Ego y Alter, es en el punto en el cual el tejido y el texto confluyen -y chocan, y se entretejen pero también se mantienen distintos- en donde la materialidad de los procesos de construcción no puede englobar ni subsumir los procesos comunicacionales humanos, los actos discursivos del propio lenguaje. ¿O será acaso que sostendremos que la historicidad humana es idéntica a la historicidad de las plantas? ¿No sería esto colapsar historicidad y temporalidad? ¿No es este riesgo un fantasma presente en todos estos proyectos postdualistas que luchan contra el excepcionalismo humano? Quizás por estas razones Latour abandonó su propio proyecto de ontologías aplanadas en favor de una reflexión en torno a diversos modos de existencia (Latour 2013).

En cualquier caso, es verdad que no hay una naturaleza humana ahistórica y única. Pero si Ego y Alter son recursos el uno del otro en su mutua constitución, ello es porque siguen manteniendo cierta coherencia, cierta independencia en la cual no se disuelven del todo en una masa indiferenciada. De allí que no sea tan fácil invocar la noción de intra-acción que Karen Barad (2007) nos ha sugerido para reemplazar la de interacción; si la segunda presupone un hibridismo que remite a purezas que esencializan a la naturaleza y a la cultura, la primera parece implicar un total colapso en el cual materia y significado terminan por colapsar en una mutua identidad.

Ello no sólo parece indeseable porque son dos dominios que, si bien no son completamente autónomos, tampoco están gobernados por los mismos procesos. Pero, de forma más importante, porque esa imagen de unidad radical termina por evocar un inmovilismo evolutivo en el cual el surgimiento de lo humano no ha implicado la articulación de una noción de mundo mucho más enriquecida. Una noción de mundo que resulta fundamental para todo proyecto normativo que pretenda rescatar las nociones de justicia y dignidad incluso si lo hace de una forma no antropocéntrica.

Vale entonces la pena señalar que la construcción material del mundo, esa inter-materialidad, implica un reconocimiento de cierta agencia en los seres vivos y de su clara capacidad de construir nichos. Son, por tanto, sujetos con mundos. Pero así como los textos no son tejidos, ni la materia es discurso ni el discurso es materia, incluso si la inter-materialidad es condición de posibilidad de la interdiscursividad, así también el ser humano construye mundos ontológicamente enriquecidos pues están investidos de significación.

Curioso juego de palabras ya que, como bien lo sabe todo aquél que haya leído El traje nuevo del Emperador, de Hans Christian Andersen, las palabras no visten porque los textos no son textiles. Pero, y 
casi en contraposición, como también nos recuerda Derrida (2008), para estar desnudo hace falta algo más que no llevar textil alguno, hace falta estar investido o revestido de normas que recubren al cuerpo y lo sumergen en la cultura como un fenómeno de significación.

A lo que voy, por tanto, es que los mundos en los cuales los humanos habitamos son plexos de significatividad pragmática (Heidegger 1962) -es decir, constituidos por agentes que actúan y que tienen fines, valores y deseos que revisten al mundo material con una capa de significación- en la cual la interdiscursividad y la intermaterialidad se imbrican pero no se disuelven la una en la otra. Curiosamente, sólo esta distinción puede evitar que lo histórico colapse en lo temporal porque la historia se funda allí donde el tiempo no es un simple fluir de momentos sino que es el acto mismo de reconocer, comprender y simbolizar nuestras deudas y dependencias para con el pasado.

Así, si bien es cierto que nunca hemos sido humanos, si eso nos remite a una noción de naturaleza humana transhistórica y asociada al humanismo del siglo XVIII -iluminado y colonial-, ello no implica que el multinaturalismo requiera claudicar de la idea de las múltiples naturalezas. Me resisto, por tanto, a subsumir a la construcción de mundos, a la construcción de cultura, como un tipo de construcción de nicho o como un mero proceso homólogo al que cualquier otro organismo es capaz de llevar a cabo. Me resisto porque los "mundos humanos" no sólo implican una agencia "en sí", una capacidad de experimentar y operar en el mundo "en sí" sino que, además, incluyen el ser un agente "para sí", el experimentar y operar en el mundo "para sí mismo". Siguiendo con el ejemplo, nosotros podemos percatarnos del hecho de nuestra desnudez y ese hecho traza las fronteras de Ego y Alter de una forma que no es tan trivial como puede parecer.

Enarbolo, por tanto, la siguiente tesis. En las disciplinas de la marginalidad todo intento de superación dialéctica, de reducción, de unificación, de subsunción, etc. de las lógicas de las disciplinas que allí confluyen y de sus infrapolíticas de la identificación y de la des-identificación es a la vez inevitable e inevitablemente político pues implica la postulación de una arquitectónica de la coherencia. Ello ocurre porque es en esas disciplinas donde las infrapolíticas emergen en su máxima tensión, con sus diversas categorizaciones asociadas.

Por lo anterior es que quiero señalar que más allá de los debates sobre el reduccionismo, el holismo, el monismo metodológico y demás, aquí se juega un proyecto de articulación de arquitecturas del conocimiento, de arquitectónicas de la coherencia, que es constitutivo de la marginalidad de ciertos saberes en los cuales no dejarán de definirse los límites de lo humano.

\section{Conclusión}

Si este texto se intitula "Naturalezas, culturas y arquitecturas disciplinarias. La infrapolítica de un debate (necesariamente) interminable" es porque pretende hacer un punto importante sobre las críticas que se dirigen hacia la biología desde los discursos que enarbolan los estudios de género, sexualidad y raza u otros semejantes que de una u otra manera critican la noción de naturaleza humana que la biología nos ofrece (Hager 1997, Schiebinger 2004). Parecería que la temporalidad que subyace a la biología evolutiva hace imposible cualquier esencialismo y, sin embargo, la cancelación de la historicidad de la propia naturaleza humana es justo la fuente de esta tensión pues implica la reducción de dicha historicidad en una mera temporalidad.

Y esa historicidad es justamente la que ignora cualquier proyecto reduccionista, holista, constructivista, etcétera en el cual se pierde de vista que, si bien nunca hemos sido humanos, sólo nosotros conocemos la desnudez. Hoy por hoy hay discursos en biología que, operando por medio de los mecanismos antiparalelos ya mencionados, amplifican las agencias mientras deshumanizan lo humano. Generan una arquitectónica de la coherencia en la cual, nuevamente, las humanidades y las ciencias sociales se vuelven marginales dentro de la marginalidad. 
Barad, K. (2007), Meeting the Universe Halfway: Quantum Physics and the Entanglement of Matter and Meaning, Durham: Duke University Press.

Derrida, J. (2008), The Animal that Therefore I Am, New York: Fordham University Press.

Foucault, M. (1970), The Order of Things. An Archaeology of the Human Sciences, New York: Random House Inc.

Foucault, M. (1972), The Archaeology of Knowledge, New York: Tavistock Publication Limited.

Giffney, N. y M. Hird (2008), Queering the Non/Human, Burlington: Ashgate Publishing Limited.

Hager, L. (1997), "Sex and Gender in Paleoanthropology", en Hager, L. (ed.), Women in Human Evolution, Nueva York: Routledge, pp. 1-28.

Haraway, D. (2007), When Species Meet, Minneapolis: University of Minnesota Press.

Heidegger, M. (1962), Being and Time, New York: Harper and Row Publishers, Inc.

Laland, K., Sterelny, K., Odling-Smee, J., Hoppitt, W. y U. Tobias (2011), "Cause and Effect in Biology Revisited: Is Mayr's Proximate-Ultimate Dichotomy Still Useful?”, Science 334: 313-325.

Latour, B. (2002), War of the Worlds: What about Peace?, Chicago: Pickly Paradigm Press.

Latour, B. (2013), An Inquiry into Modes of Existence, Cambridge: Harvard University Press.

Marder, M. (2014), Phenomena-Critique-Logos: The Project of Critical Phenomenology, New York: Rowman \& Littlefield International.

Marks, J. (2011), The Alternative Introduction to Biological Anthropology, Oxford: Oxford University Press.

Maynard-Smith, J. y S. Eörs (2000), The Origins of Life. From the Birth of Life to the Origin of Language, Oxford: Oxford University Press.

Odling-Smee, J. y L. Kevin (2011), "Ecological Inheritance and Cultural Inheritance: What are They and How Do They Differ?”, Biological Theory 6: 220-230.

Schiebinger, L. (2004), Nature's Body. Gender in the Making of Modern Science, New York: Rutgers University Press.

Schultz, E. (2015), "La construcción de nicho y el estudio de los cambios de cultura en antropología: desafíos y perspectivas", Interdisciplina 3(5): 131-160.

Wilson, E.O. (2000), Sociobiology. The New Synthesis, Cambridge, MA: The Belknap Press of Harvard University Press. 